Pacific Journal of Mathematic 


\title{
SOME REPRODUCING KERNELS FOR THE UNIT DISK
}

\author{
G. S. INNIS, JR.
}

Introduction. Let $S(t)$ denote the class of functions $\varphi$ analytic in the unit disk $U$ with center 0 and satisfying

$$
\int_{U} \int|\varphi(z)|\left(1-|z|^{2}\right)^{t} d x d y<\infty \quad(z=x+i y)
$$

for $t$ real. In this paper we shall prove that for $\lambda$ and $\nu$ properly restricted, $|\zeta|<1$ and $\varphi \in S(t)$, the following formulas are valid:

$$
\varphi(\zeta)=\frac{(\lambda+1)^{\nu}}{\Gamma(\nu) \pi} \int_{J} \int \frac{\varphi(z)\left(1-|z|^{2}\right)^{\lambda}}{(1-\bar{z} \zeta)^{\lambda+2}} \ln ^{\nu-1}\left(\frac{1-\bar{z} \zeta}{1-|z|^{2}}\right) d x d y,
$$

and

(3) $\varphi^{(m)}(\zeta)=\frac{\lambda+1}{\pi} \iint \bar{z}^{m} \frac{\varphi(z)\left(1-|z|^{2}\right)^{\lambda}}{(1-\bar{z} \zeta)^{\lambda+2+m}} \sum_{i=0}^{m} a_{i} l n^{\nu-1-i}\left(\frac{1-\bar{z} \zeta}{1-|z|^{2}}\right) d x d y$, where the $a_{i}$ are suitably chosen constants (with respect to $\varphi$ and the variables $z$ and $\zeta$ ). Finally, if

$$
\begin{aligned}
F_{n}(\zeta, \nu, \lambda)= & \frac{(-1)^{n+1}}{\pi} \iint \frac{\varphi(z)\left(1-|z|^{2}\right)^{\lambda}}{\bar{z}^{n}(1-\bar{z} \zeta)^{\lambda+2-n}} \\
& \cdot\left[\frac{(\lambda+1)^{\nu-1}}{\Gamma(\nu+n-1)} l n^{\nu+n-2}\left(\frac{1-\bar{z} \zeta}{1-|z|^{2}}\right)\right. \\
& \left.+\frac{1}{\Gamma(n)} \ln ^{n-1}\left(\frac{1-\bar{z} \zeta}{1-|z|^{2}}\right)\right] d x d y,
\end{aligned}
$$

then $F_{n}(\zeta, \nu, \lambda)$ has the property that

$$
\frac{d^{n}}{d \zeta^{n}} F_{n}(\zeta, \nu, \lambda)=\varphi(\zeta)
$$

Formula (2) reduces to the well known results of Ahlfors [1] and Bergman [2] for particular choices of the parameters $t, \lambda$, and $\nu$. The author is indebted to Professor Ahlfors for suggesting this problem.

Notation. Define

$$
\begin{aligned}
N(z, \lambda) & =\left(1-|z|^{2}\right)^{\lambda}, \\
D(z, \zeta, \lambda) & =(1-\bar{z} \zeta)^{\lambda}, \\
L(z, \zeta, \nu) & =\ln ^{\nu-1}\left(\frac{1-\bar{z} \zeta}{1-|z|^{2}}\right)
\end{aligned}
$$

Received May 15, 1963. This work was done while the author was a NAS-NRC Postdoctoral Fellow. 
where the principal values of the functions on the right are used.

Reproducing Kernels. In this section we shall prove

THEOREM 1. If $\varphi \in S(t)$ for some $t$, then

(a) for $\operatorname{Re} \nu \geqq 1$ and $\operatorname{Re} \lambda>t$, (2) is satisfied and

(b) for $\operatorname{Re} \nu=1$ and $\operatorname{Re} \lambda \geqq t$, (2) is satisfied.

REMARKS. If

$$
K_{1}(z, \zeta, \nu, \lambda)=\frac{(\lambda+1)^{\nu}}{\Gamma(\nu) \pi} N(z, \lambda) D(z, \zeta,-\lambda,-2) L(z, \zeta, \nu),
$$

then because $|z|<1,|\zeta|<1$ and principal values were used in defining $N, D$ and $L, K_{1}$ is unambiguously defined. Thus (2) can be written

$$
\varphi(\zeta)=\int_{\sigma} \int \varphi(z) K_{1}(z, \zeta, \nu, \lambda) d x d y .
$$

Also, if $\varphi \in S(t)$ and $\varphi \not \equiv 0$, then $t>-1$ as is easily seen by considering (1) in polar coordinates.

The proof of Theorem 1 will be preceded by the statement and proof of three lemmas.

LEMMA 1. For $\varphi \in S(t)$, and for $R e \lambda \geqq t$, (1) implies

$$
\lim _{r \rightarrow 1}\left(1-r^{2}\right)^{R e \lambda+1} \int_{0}^{2 \pi}\left|\varphi\left(r e^{i \theta}\right)\right| d \theta=0 .
$$

Proof. If $f(r)=\int_{0}^{2 \pi}\left|\varphi\left(r e^{i \theta}\right)\right| d \theta$, then $f$ is a nondecreasing function of $r$ for $0<r<1$ (the trivial case of $\varphi \equiv 0$ is excluded in the sequel). Suppose now that $\lim \sup \left(1-r^{2}\right)^{R e \lambda+1} f(r)=a>0$ ( $a$ may be infinite). Let $0<b<a$. Then there exists a sequence $\left\{r_{i}\right\}$ of real numbers, $0<r_{i-1}<r_{i}<1$, converging to 1 such that $f(r) \geqq b\left(1-r_{i}^{2}\right)^{-(R e \lambda+1)}$ for $r>r_{i}$ and $1-r_{i}^{2}<\left(1-r_{i-1}^{2}\right) / 2$. Then (1) becomes

$$
\begin{aligned}
& \int_{0}^{1} \int_{0}^{2 \pi} r\left(1-r^{2}\right)^{R e \lambda}\left|\varphi\left(r e^{i \theta}\right)\right| d r d \theta \geqq \sum_{i=2}^{\infty} f\left(r_{i-1}\right) \int_{r_{i-1}}^{r_{i}} r\left(1-r^{2}\right)^{R e \lambda} d r \\
& =\sum_{i=2}^{\infty} \frac{b}{R e \lambda+1}\left[1-\left(\frac{1-r_{i}^{2}}{1-r_{i-1}^{2}}\right)^{R e \lambda+1}\right] \\
& \quad \geqq \sum_{i=2}^{\infty} \frac{b}{R e \lambda+1} 1^{i}\left[1-\left(\frac{1}{2}\right)^{R e \lambda+1}\right]=\infty .
\end{aligned}
$$

This contradiction implies

$$
\lim _{r \rightarrow 1}\left(1-r^{2}\right)^{R e \lambda+1} \int_{0}^{2 \pi}\left|\varphi\left(r e^{i \theta}\right)\right| d \theta=0 .
$$


LEMMA 2. If $\varphi \in S(t)$ for some $t$, Re $>t$ and Reע $\geqq 1$, then

$$
\int_{U} \int \varphi(z) K_{1}(z, \zeta, \nu, \lambda) d x d y=\int_{\sigma} \int \varphi(z) K_{1}(z, \zeta, \nu+1, \lambda) d x d y .
$$

Proof. Let $K_{1}(\nu)=K_{1}(z, \zeta, \nu, \lambda)$. Then

$$
K_{1}(\nu)=\left[K_{1}(\nu)-K_{1}(\nu+1)\right]+K_{1}(\nu+1)
$$

and if

$$
f(z, \zeta, \nu, \lambda)=\frac{(\lambda+1)^{\nu}}{\Gamma(\nu+1) \pi} \frac{\varphi(z)}{z-\zeta} N(z, \lambda+1) D(z, \zeta,-\lambda-1) L(z, \zeta, \nu+1),
$$

then

$$
\frac{\partial f}{\partial \bar{z}}=\left(K_{1}(\nu)-K_{1}(\nu+1)\right) \varphi(z) .
$$

We are, therefore, in a position to apply Green's formula since the singularity of $f$ at $z=\zeta$ is only apparent $\left(\lim _{z \rightarrow \zeta}(z-\zeta)^{-1} L(z, \zeta, \nu+1)=0\right)$. Thus for $0<r<1$,

$$
\begin{aligned}
\int_{|z|<r} \int \varphi(z) K_{1}(\nu) d x d y= & \frac{1}{2 i} \int_{|z|=r} f(z, \zeta, \nu, \lambda) d z \\
& +\int_{|z|<r} \int \varphi(z) K_{1}(\nu+1) d x d y,
\end{aligned}
$$

and the lemma will be proved if we establish that the line integral in (7) vanishes as $r \rightarrow 1$. To show that this is the case, let $\varepsilon>0$ and $t+\varepsilon<\operatorname{Re} \lambda$. Then

$$
\begin{aligned}
& I_{r}=\frac{1}{2 i} \int_{|z|=r} f(z, \zeta, \nu, \lambda) d z \\
& =C \int_{0}^{2 \pi} \frac{\varphi\left(r e^{i \theta}\right)}{r e^{i \theta}-\zeta} N(r, \lambda+1) D\left(r e^{i \theta}, \zeta,-\lambda-1\right) L\left(r e^{i \theta}, \zeta, \nu+1\right) r e^{i \theta} d \theta,
\end{aligned}
$$

and for $r$ near 1 ,

$$
\left|I_{r}\right| \leqq C_{1}\left(1-r^{2}\right)^{R e \lambda+1-\varepsilon / 2} \int_{0}^{2 \pi}\left|\varphi\left(r e^{i \theta}\right)\right| d \theta
$$

where the factor $\left(1-r^{2}\right)^{\varepsilon / 2}$ was used to suppress the logarithm near $r=1$. On applying Lemma 1 in (9) we get

$$
\left|I_{r}\right| \leqq C_{2}\left(1-r^{2}\right)^{\varepsilon / 2},
$$

and the result follows.

LeMma 2'. Lemma 2 is valid for $\operatorname{Re} \lambda \geqq t$ if $\operatorname{Re\nu }=1$. 
Proof. The proof of this lemma is similar to that of Lemma 2 except that the factor of $\left(1-r^{2}\right)^{\varepsilon / 2}$ is not needed to suppress the logarithm and, therefore, the range of $\lambda$ can be extended.

LEMmA 3. If Reע $\geqq k, R e \lambda>-1$ and $p$ is a positive integer, then

$$
\begin{aligned}
& \int_{0}^{1} r^{2 p-1} N(r, \lambda) L(r, 0, \nu-k+1) d r \\
& \quad=\sum_{i=0}^{p-1}(-1)^{i}\left(\begin{array}{c}
p-1 \\
i
\end{array}\right) \frac{\Gamma(\nu-k+1)}{2(\lambda+i+1)^{\nu-k+1}} .
\end{aligned}
$$

Proof. Induction on $p$ will be used. If $p=1,(10)$ reads

$$
\int_{0}^{1} r N(r, \lambda) L(r, 0, \nu-k+1) d r=\frac{\Gamma(\nu-k+1)}{2(\lambda+1)^{\nu-k+1}} .
$$

Substituting

$$
t=(\lambda+1) L(r, 0,2), \quad d t=(\lambda+1) \frac{2 r}{1-r^{2}} d r
$$

in the left hand side, we get

$$
\int_{0}^{1} r N(r, \lambda) L(r, 0, \nu-k+1) d r=\frac{1}{2(\lambda+1)^{\nu-k+1}} \int_{0}^{\infty} e^{-t} t^{\nu-k} d t
$$

where the path of integration in the right hand member is the half line through the origin inclined at the angle arg $(\lambda+1)$. That integral is $\Gamma(\nu-k+1)$, and the result is established for $p=1$. Suppose that (10) has been proved for $p-1$. The left hand side of (10) can be written in the form

$$
\begin{gathered}
\int_{0}^{1} r^{2 p-3} N(r, \lambda) L(r, 0, \nu-k+1) d r-\int_{0}^{1} r^{2 p-3} N(r, \lambda+1) L(r, 0, \nu-k+1) d r \\
=\frac{\Gamma(\nu-k+1)}{2(\lambda+1)^{\nu-k+1}}+\sum_{i=1}^{p-2}(-1)^{i}\left[\left(\begin{array}{c}
p-2 \\
i+1
\end{array}\right)+\left(\begin{array}{c}
p-2 \\
i
\end{array}\right)\right] \frac{\Gamma(\nu-k+1)}{2(\lambda+1+i)^{\nu-k+1}} \\
\quad+(-1)^{p-1} \frac{\Gamma(\nu-k+1)}{2(\lambda+p)^{\nu-k+1}}=\sum_{i=0}^{p-1}(-1)^{i}\left(\begin{array}{c}
p-1 \\
i
\end{array}\right) \frac{\Gamma(\nu-k+1)}{2(\lambda+i+1)^{\nu-k+1}} .
\end{gathered}
$$

Proof of Theorem 1. This proof will be accomplished by showing that the $m$ th derivative of $\varphi$ evaluated at 0 is given by the $m$ th derivative of (2) evaluated at 0 . Induction will be used.

It is clear that (1) implies the absolute convergence of (2), and that if $R e \lambda$ is large enough, differentiation with respect to $\zeta, \lambda$, and $\nu$ will commute with integration. Differentiating (2) $m$ times with respect to $\zeta$, one gets 


$$
\begin{aligned}
\varphi^{(m)}(\zeta)= & \frac{\lambda+1}{\pi} \int_{\sigma} \int_{\bar{z}^{m}} \varphi(z) N(z, \lambda) D(z, \zeta,-\lambda-2-m) \\
& \sum_{i=0}^{m} a_{i} L(z, \zeta, \nu-i) d x d y
\end{aligned}
$$

if $R e \nu \geqq m+1$ and the $\alpha_{i}$ are properly chosen constants.

Let $F(\zeta)=\int_{V} \int \varphi(z) K_{1}(\nu) d x d y$. Then $F(0)=\int_{\sigma} \int \varphi(z) K_{1}(z, 0, \nu, \lambda) d x d y$ which by (1) can be written

$$
\begin{aligned}
F(0) & =\frac{(\lambda+1)^{\nu}}{\Gamma(\nu) \pi} \int_{0}^{1} r N(r, \lambda) L(r, 0, \nu) d r \int_{0}^{2 \pi} \varphi\left(r e^{i \theta}\right) d \theta \\
& =\frac{2(\lambda+1)^{\nu}}{\Gamma(\nu)} \varphi(0) \int_{0}^{1} r N(r, \lambda) L(r, 0, \nu) d r .
\end{aligned}
$$

By Lemma 3 this last integral is $\Gamma(\nu) / 2(\lambda+1)^{\nu}$, and the desired result follows.

Suppose now that $R e \nu>1$. Because of a complication in the inductive hypothesis, it will also be necessary to show that $F^{\prime}(0)=$ $\varphi^{\prime}(0)$. Notice, however, that if we differentiate $F$ with respect to $\zeta$ two terms arise, and in one of these the exponent of $\ln$ is $\nu-2$. If $R e \nu<2$, this would cause trouble. This difficulty is avoided if we first apply Lemma 2 to $F$ to write it in a form for which $R e \nu \geqq 2$. Then

$$
\begin{aligned}
F^{\prime}(0)= & \frac{(\lambda+1)^{\nu}}{\Gamma(\nu) \pi} \iint \bar{z} \varphi(z) N(z, \lambda) \\
& {[(\lambda+2) L(z, 0, \nu)-(\nu-1) L(z, 0, \nu-1)] d x d y . }
\end{aligned}
$$

By splitting this into two integrals and proceeding just as above, we derive

$$
F^{\prime}(0)=\varphi^{\prime}(0) \text {. }
$$

Suppose now that it has been established that $F^{(p-1)}(0)=\varphi^{(p-1)}(0)$. Use Lemma 2 to write $F$ in a form for which $R e \nu \geqq p+1$.

Let the following be taken as the inductive hypothesis:

$$
\begin{gathered}
F^{(p-1)}(0)=\varphi^{(p-1)}(0), \\
a_{0}+\sum_{i=1}^{p-1} a_{i} \frac{(\lambda+1)^{i}}{(\nu-1)(\nu-2) \cdots(\nu-i)}=(p-1) !,
\end{gathered}
$$

and

$$
a_{0}+\sum_{i=1}^{p-1} a_{i} \frac{(\lambda+k)^{i}}{(\nu-1)(\nu-2) \cdots(\nu-i)}=0
$$

for $k=2,3, \cdots, p$. When $p=2$, (12a) was proved above. In this 
case $a_{0}=\lambda+2$ and $a_{1}=-(\nu-1)$ so that both $(12 b)$ and $(12 \mathrm{c})$ are satisfied. Consider now $F^{(p)}(0)$ when $F^{(p-1)}(\zeta)$ is given by the right hand side of (11) with $m=p-1$.

$$
\begin{gathered}
F^{(p)}(0)=\frac{(\lambda+1)^{\nu}}{\Gamma(\nu) \pi} \int_{U} \int_{z^{p}} \varphi(z) N(z, \lambda) \\
{\left[(\lambda+1+p) \sum_{i=0}^{p-1} a_{i} L(z, 0, \nu-i)-\sum_{i=0}^{p-1} a_{i}(\nu-i) L(z, 0, \nu-i-1)\right] d x d y .}
\end{gathered}
$$

After some algebra (13) becomes

$$
\begin{aligned}
F^{(p)}(0)= & \frac{2(\lambda+1)^{\nu}}{p ! \Gamma(\nu)} \phi^{(p)}(0)\left[b_{0} \frac{\Gamma(\nu)}{2(\lambda+1)^{\nu}}-b_{1}\left(\begin{array}{c}
p \\
1
\end{array}\right) \frac{\Gamma(\nu)}{2(\lambda+2)^{\nu}}\right. \\
& \left.+\cdots(-1)^{p} b_{p} \frac{\Gamma(\nu)}{2(\lambda+p+1)^{\nu}}\right]
\end{aligned}
$$

where

$$
\begin{aligned}
b_{0}= & a_{0}(\lambda+1+p)+\frac{\lambda+1}{\nu-1}\left[a_{1}(\lambda+1+p)-a_{0}(\nu-1)\right] \\
& +\frac{(\lambda+1)^{2}}{(\nu-1)(\nu-2)}\left[a_{2}(\lambda+1+p)-a_{1}(\nu-2)\right] \\
& +\cdots-a_{p-1}(\nu-p) \frac{(\lambda+1)^{p}}{(\nu-1)(\nu-2) \cdots(\nu-p)} \\
= & (\lambda+1+p)(p-1) !-(\lambda+1)(p-1) ! \\
= & p ! \quad \text { by }(12 b)
\end{aligned}
$$

and

$$
\begin{aligned}
b_{k}= & a_{0}(\lambda+1+p)+\frac{\lambda+k+1}{\nu-1}\left[a_{1}(\lambda+1+p)-a_{0}(\nu-1)\right] \\
& +\frac{(\lambda+k+1)^{2}}{(\nu-1)(\nu-2)}\left[a_{2}(\lambda+1+p)-a_{1}(\nu-2)\right] \\
& +\cdots-a_{p-1}(\nu-p) \frac{(\lambda+k+1)^{p}}{(\nu-1)(\nu-2) \cdots(\nu-p)} \\
= & (\lambda+1+p) 0+(\lambda+k+1) 0=0 \text { by (12c) for }
\end{aligned}
$$

$k=2,3, \cdots, p$. It follows immediately that

$$
F^{(p)}(0)=\varphi^{(p)}(0)
$$

as was to be shown.

The case $R e \nu=1, R e \lambda \geqq t$ is treated as above except that Lemma $2^{\prime}$ is used in place of Lemma 2. The proof is omitted.

REMARKs. Notice that in proving Theorem 1 we have also"established 
that (11) is a correct formula for the $m$ th derivative of $\varphi$.

As mentioned above we are also at liberty to differentiate (2) with respect to $\nu$ and $\lambda$. It is readily verified that differentiating (2) with respect to $\lambda$ and using the results of Theorem 1 yields

$$
\varphi(\zeta)=\int_{\sigma} \int \varphi(z) K_{1}(\nu+1) d x d y
$$

which is nothing new. However, differentiating (2) with respect to $\nu$ and using Theorem 1 we derive the new formula,

$$
\begin{gathered}
\varphi(\zeta)=\frac{(\lambda+1)^{\nu}}{\Gamma^{\prime}(\nu) \pi-\ln (\lambda+1) \Gamma(\nu) \pi} \int_{\sigma} \int \varphi(z) N(z, \lambda) D(z, \zeta,-\lambda-2) \\
L(z, \zeta, \nu) \ln (L(z, \zeta, 2)) d x d y .
\end{gathered}
$$

The integral in (14) is absolutely convergent in spite of the apparent difficulties with $\ln (L)$. Further derivations with respect to $\zeta, \nu$, and $\lambda$ are, of course, possible.

An interesting formula results from (11) for the case in which $\lambda$ is an integer and $\nu=1$. Here, $a_{0}=\Gamma(n+m+1) / \Gamma(n+1)$ and the rest of the $a$ 's are zero. The $\theta$ integral is

$$
\int_{0}^{2 \pi}\left(r e^{-i \theta}\right)^{m} \frac{\varphi\left(r e^{i \theta}\right)}{\left(1-r e^{-i \theta} \zeta\right)^{m+n+2}} d \theta=2 \pi \frac{r^{2 m}}{(m+n+1) !}\left[z^{n+2} \varphi(z)\right]_{z=r 2 \zeta}^{(m+n+1)},
$$

and (11) becomes

$$
\varphi^{(m)}(\zeta)=\frac{2}{n !} \int_{0}^{1} r^{2 m+1}\left(1-r^{2}\right)^{n}\left[z^{n+2} \varphi(z)\right]_{z=r 2 \zeta}^{(m+n+1)} d r .
$$

This expression is readily checked for $\varphi(z)=z^{k}$ and, thereby, for any $\varphi \in S(n)$.

Primative Kernels. In this section we shall prove

TheOREM 2. If $\varphi \in S(t)$ and

$$
\begin{aligned}
K_{2}^{n}(z, \zeta, \nu, \lambda)=\frac{(-1)^{n+1}}{\bar{z}^{n} \pi} N(z, \lambda) D(z, \zeta,-\lambda-2+n) \\
\quad\left[\frac{(\lambda+1)^{\nu-1}}{\Gamma(\nu+n-1)} L(z, \zeta, \nu+n-1)+\frac{1}{\Gamma(n)} L(z, \zeta, n)\right],
\end{aligned}
$$

then for $\operatorname{Re} \nu=2$ and $\operatorname{Re} \lambda \geqq t$ or $\operatorname{Re} \nu \geqq 2$ and $\operatorname{Re} \lambda>t$,

$$
F_{n}(\zeta, \nu, \lambda)=\int_{\sigma} \int \varphi(z) K_{2}^{n}(z, \zeta, \nu, \lambda) d x d y
$$

has the property that $F_{n}^{(n)}(\zeta, \nu, \lambda)=\varphi(\zeta)$ (differentiation is with respect to $\zeta)$. If $R e \lambda \geqq t$ and $\nu=1$, then 


$$
H_{n}(\zeta, \lambda)=\iint \varphi(z) K_{2}^{n}(z, \zeta, 1, \lambda) d x d y
$$

has the property that $H_{n}^{(n)}(\zeta, \lambda)=2 \varphi(\zeta)$.

Proof. The proof will be by induction. Consider $F_{1}(\zeta)$. To differentiate under the integral sign in (15) it is sufficient to show that the given and resulting integrals are absolutely convergent. However,

$$
\int_{\sigma} \int\left|\varphi(z) K_{2}^{1}(z, \zeta, \nu, \lambda)\right| d x d y=\int_{|z| \leqq r} \int+\int_{r<|z|<1} \int .
$$

The integral over the annulus offers no difficulty and for small $r$,

$$
\left|\varphi(z) K_{2}^{1}(z, \zeta, \nu, \lambda)\right| \leqq C \frac{1}{r}
$$

where $C$ is constant. Thus

$$
\int_{|z| \leqq r} \int\left|\varphi(z) K_{2}^{1}(z, \zeta, \nu, \lambda)\right| d x d y \leqq 2 \pi r C .
$$

Because $R e \nu \geqq 2$, all of the integrals occurring after differentiation are absolutely convergent and, hence,

$$
\begin{aligned}
F_{1}^{\prime}(\zeta, \nu, \lambda) & =\int_{\sigma} \rho \varphi(z) \frac{\partial}{\partial \zeta} K_{2}^{1}(z, \zeta, \nu, \lambda) d x d y \\
& =\int_{\sigma} \int \varphi(z)\left[K_{1}(\nu)+K_{1}(1)-K_{1}(\nu-1)\right] d x d y \\
& =\varphi(\zeta) .
\end{aligned}
$$

Similarly $H_{1}^{\prime}(\zeta, \lambda)=2 \varphi(\zeta)$ and thus

$$
H_{1}(\zeta, \lambda)=2 F_{1}(\zeta, \nu, \lambda)+C \text {. }
$$

Suppose now that it has been established that for some $n \geqq 2$,

(a) $F_{n-1}(\zeta, \nu, \lambda)$ is an $(n-1)$ st primative and

(b) $H_{n-1}(\zeta, \lambda)=2 F_{n-1}(\zeta, \nu, \lambda)+P(\zeta, \nu, \lambda)$ where

$P$ is a polynomial of degree $n-2$ in $\zeta$. The absolute convergence of the needed integrals can be established as above. Therefore, from (15) we get

$$
\begin{aligned}
F_{n}^{\prime}(\zeta, \nu, \lambda)= & \frac{(-1)^{n+1}}{\pi} \int_{\sigma} \int_{\frac{\varphi(z)}{\bar{z}^{n-1}}} N(z, \lambda) D(z, \zeta,-\lambda-1+n) \\
& {\left[(\lambda+2-n) \frac{(\lambda+1)^{\nu-1}}{\Gamma(\nu+n-1)} L(z, \zeta, \nu+n-1)\right.} \\
& +(\lambda+2-n) \frac{1}{\Gamma(n)} L(z, \zeta, n) \\
& -\frac{(\lambda+1)^{\nu-1}}{\Gamma(\nu+n-2)} L(z, \zeta, \nu+n-2) \\
& \left.-\frac{1}{\Gamma(n-1)} L(z, \zeta, n-1)\right] d x d y .
\end{aligned}
$$


The last two terms in this square bracket yield $F_{n-1}(\zeta, \nu, \lambda)$. Now let us add and subtract $2(\lambda+2-n) L(z, \zeta, n-1) /[(\lambda+1) \Gamma(n-1)]$ to the first two terms to write them as

$$
\begin{aligned}
\frac{\lambda+2}{\lambda+}-1 & {\left[\frac{(\lambda+1)^{\nu-1}}{\Gamma(\nu+n-2)} L(z, \zeta, \nu+n-2)+\frac{1}{\Gamma(n-1)} L(z, \zeta, n-1)\right.} \\
& +\frac{(\lambda+1)^{\nu-1}}{\Gamma(\nu+n-2)} L(z, \zeta, \nu+n-2)+\frac{1}{\Gamma(n-1)} L(z, \zeta, n-1) \\
& \left.-\frac{2}{\Gamma(n-1)} L(z, \zeta, n-1)\right]
\end{aligned}
$$

where the first term comes from the first term of (17) with $\nu$ replaced by $\nu+1$ and the third term comes from the second term of (17) with $\nu=2$. Thus (17) yields

$$
\begin{aligned}
F_{n}^{\prime}(\zeta, \nu, \lambda)= & F_{n-1}(\zeta, \nu, \lambda)-\frac{\lambda+2-n}{\lambda+1}\left[F_{n-1}(\zeta, \nu+1, \lambda)\right. \\
& \left.+F_{n-1}(\zeta, 2, \lambda)-H_{n-1}(\zeta, \lambda)\right] \\
= & F_{n-1}(\zeta, \nu, \lambda)+Q(\zeta, \nu, \lambda)
\end{aligned}
$$

where $Q$ is a polynomial of degree $(n-2)$ in $\zeta$.

To complete the inductive argument, it is necessary to show that $H_{n}^{\prime}(\zeta, \lambda)=2 F_{n-1}(\zeta, \nu, \lambda)+P(\zeta, \nu, \lambda)$.

$$
\begin{aligned}
H_{n}^{\prime}(\zeta, \lambda) & =2(-1)^{n+1} \int_{U} \frac{\varphi(z)}{\bar{z}^{n-1}} N(z, \lambda) D(z, \zeta,-\lambda-1+n) \\
& {\left[\frac{\lambda+2-n}{\Gamma(n)} L(z, \zeta, n)-\frac{1}{\Gamma(n-1)} L(z, \zeta, n-1)\right] d x d y . }
\end{aligned}
$$

Using the same techniques as above, the square brackets can be written

$$
\begin{aligned}
& \frac{\lambda+2-n}{\lambda+1}\left[\frac{(\lambda+1)^{\nu-1}}{\Gamma(\nu+n-2)} L(z, \zeta, \nu+n-2)+\frac{1}{\Gamma(n-1)} L(z, \zeta, n-1)\right] \\
& \quad-\left(\frac{\lambda+2-n}{\lambda+1}+1\right) \frac{1}{\Gamma(n-1)} L(z, \zeta, n-1)
\end{aligned}
$$

where $\nu=2$ in the first term. On placing this expression in (18), we get

$$
H_{n}^{\prime}(\zeta, \lambda)=-2\left(\frac{\lambda+2-n}{\lambda+1}\right) F_{n-1}(\zeta, 2, \lambda)+\left(\frac{\lambda+2-n}{\lambda+1}+1\right) H_{n-1}(\zeta, \lambda) .
$$

By the inductive hypothesis, $H_{n-1}(\zeta, \lambda)=2 F_{n-1}(\zeta, \nu, \lambda)+R(\zeta, \nu, \lambda)$ where $R$ is of degree $(n-2)$ in $\zeta$. We have then that

$$
H_{n}^{\prime}(\zeta, \lambda)=2 F_{n-1}(\zeta, \nu, \lambda)+P(\zeta, \nu, \lambda)
$$

where $P$ is of degree $(n-2)$ in $\zeta$. This proves Theorem 2 . 
It is interesting to note that $F_{n}$ and $H_{n}$ depend analytically on $\nu$ and $\lambda$ and are not necessarily constants (with respect to these two variables).

It is easy to prove

THEOREM 3. If (a) $\varphi \in S(R e \lambda)$ and has a zero of order at least $n$ at 0 , (b) either $\lambda$ is not an integer or $\lambda$ is an integer greater than $n-2$, (c)

$$
K_{3}^{n}=\frac{\lambda+1}{\pi} \frac{\Gamma(\lambda+3-n)}{\Gamma(\lambda+3)} \bar{z}^{-n} N(z, \lambda) D(z, \zeta,-\lambda-2+n)
$$

and (d)

$$
G_{n}(\zeta)=\int_{\sigma} \int \varphi(z) K_{3}^{n}(z, \zeta, \lambda) d x d y
$$

then

$$
G_{n}^{(n)}(\zeta)=\varphi(\zeta)
$$

The conditions imposed on $\lambda$ are sufficient to guarantee that the integral (19) converges absolutely. The proof of the theorem is just a matter of differentiating and is omitted. If, however, $\varphi \in S(\operatorname{Re} \lambda)$, then for each positive integer $n, z^{n} \varphi(z)$ is also in $S(R e \lambda)$, and, therefore, if we define

$$
E_{n}(\zeta)=\int_{U} z^{n} \varphi(z) K_{3}^{n}(z, \zeta, \lambda) d x d y,
$$

$E_{n}(\zeta)$ is well defined, absolutely convergent and has the property that

$$
E_{n}^{(n)}(\zeta)=\zeta^{n} \varphi(\zeta)
$$

The simplicity of (20) may make it more useful then either (15) or (16) in some cases.

\section{BIBLIOGRAPHY}

1. L. V. Ahlfors, Some remarks on Teichmüller's space of Riemann surfaces, Ann. of Math., 74 (1961), 176.

2. Z. Nehari, Conformal Mapping, McGraw-Hill, 1952, p. 252.

HARVARD UNIVERSITY 


\section{PACIFIC JOURNAL OF MATHEMATICS}

\section{EDITORS}

Robert Osserman

Stanford University

Stanford, California

M. G. Arsove

University of Washington

Seattle 5 , Washington
J. DugundjI

University of Southern Califorma: Los Angeles 7, California

Lowell J. Paige

University of California

Los Angeles 24, California

\section{ASSOCIATE EDITORS}

E. F. BECKENBACH

B. H. NeumanN

F. WOLF

K. YosIDA

\section{SUPPORTING INSTITUTIONS}

UNIVERSITY OF BRITISH COLUMBIA

CALIFORNIA INSTITUTE OF TECHNOLOGY

UNIVERSITY OF CALIFORNIA

MONTANA STATE UNIVERSITY

UNIVERSITY OF NEVADA

NEW MEXICO STATE UNIVERSITY

OREGON STATE UNIVERSITY

UNIVERSITY OF OREGON

OSAKA UNIVERSITY

UNIVERSITY OF SOUTHERN CALIFORNIA
STANFORD UNIVERSITY

UNIVERSITY OF TOKYO

UNIVERSITY OF UTAH

WASHINGTON STATE UNIVERSITY

UNIVERSITY OF WASHINGTON

AMERICAN MATHEMATICAL SOCIETY CALIFORNIA RESEARCH CORPORATION SPACE TECHNOLOGY LABORATORIES NAVAL ORDNANCE TEST STATION 


\section{Pacific Journal of Mathematics}

\section{Vol. 14, No. 1 \\ May, 1964}

Richard Arens, Normal form for a Pfaffian .........................

Charles Vernon Coffman, Non-linear differential equations on cones in Banach

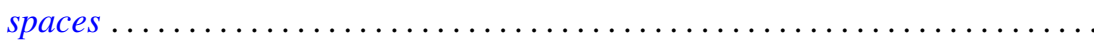

Ralph DeMarr, Order convergence in linear topological spaces ..............

Peter Larkin Duren, On the spectrum of a Toeplitz operator ................

Robert E. Edwards, Endomorphisms of function-spaces which leave stable all

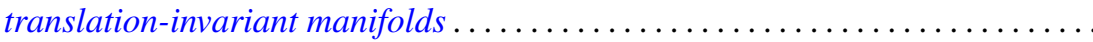

Erik Maurice Ellentuck, Infinite products of isols . . . . . . . . . . . . . . . . 49

William James Firey, Some applications of means of convex bodies . . . . . . . . 53

Haim Gaifman, Concerning measures on Boolean algebras ............. 61

Richard Carl Gilbert, Extremal spectral functions of a symmetric operator. . . . . . 75

Ronald Lewis Graham, On finite sums of reciprocals of distinct nth powers ..... 85

Hwa Suk Hahn, On the relative growth of differences of partition functions ...... 93

Isidore Isaac Hirschman, Jr., Extreme eigen values of Toeplitz forms associated

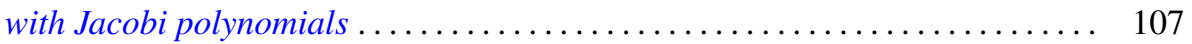

Chen-jung Hsu, Remarks on certain almost product spaces . . . . . . . . . . . 163

George Seth Innis, Jr., Some reproducing kernels for the unit disk . . . . . . . . . 177

Ronald Jacobowitz, Multiplicativity of the local Hilbert symbol . . . . . . . . . . . 187

Paul Joseph Kelly, On some mappings related to graphs ................. 191

William A. Kirk, On curvature of a metric space at a point . . . . . . . . . . . . 195

G. J. Kurowski, On the convergence of semi-discrete analytic functions . . . . . . . 199

Richard George Laatsch, Extensions of subadditive functions . . . . . . . . . . . 209

V. Marić, On some properties of solutions of $\Delta \psi+A\left(r^{2}\right) X \nabla \psi+C\left(r^{2}\right) \psi=0 \ldots 217$

William H. Mills, Polynomials with minimal value sets . . . . . . . . . . . 225

George James Minty, Jr., On the monotonicity of the gradient of a convex

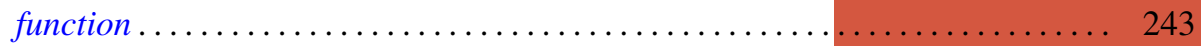

George James Minty, Jr., On the solvability of nonlinear functional equations of 'monotonic' type ................................... 249

J. B. Muskat, On the solvability of $x^{e} \equiv e(\bmod p) \ldots \ldots \ldots \ldots \ldots \ldots \ldots \ldots . \ldots \ldots$

Zeev Nehari, On an inequality of $P . R$. Bessack ................... 261

Raymond Moos Redheffer and Ernst Gabor Straus, Degenerate elliptic

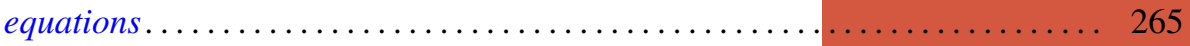

Abraham Robinson, On generalized limits and linear functionals . . . . . . . . . 269

Bernard W. Roos, On a class of singular second order differential equations with a

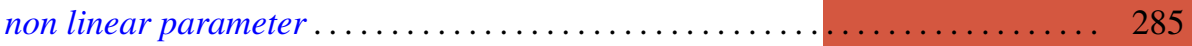

Tôru Saitô, Ordered completely regular semigroups . . . . . . . . . . . . . . . . 295

Edward Silverman, A problem of least area ....................... 309

Robert C. Sine, Spectral decomposition of a class of operators . . . . . . . . . 333

Jonathan Dean Swift, Chains and graphs of Ostrom planes . . . . . . . . . . . 353

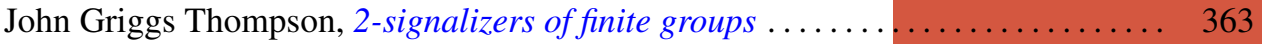

Harold Widom, On the spectrum of a Toeplitz operator . . . . . . . . . . . . . 365 